\title{
Towards fuzzy relational Galois connections between fuzzy T-digraphs
}

\author{
I.P. Cabrera, P. Cordero, E. Muñoz-Velasco, M. Ojeda-Aciego \\ Dept. Matemática Aplicada. Universidad de Málaga. Spain \\ \{ipcabrera, pcordero, ejmunoz, aciego\}@uma.es
}

\begin{abstract}
In this paper, we give the first steps towards a formal definition of fuzzy relational Galois connection between fuzzy sets with arbitrary fuzzy transitive relations (fuzzy T-digraphs), where the two components of the connection are fuzzy relations. To this end we consider, on the one hand, our definition of relational Galois connection between T-digraphs in the crisp case; and, on the other hand, our definition of fuzzy relational Galois connection between fuzzy preorders. We compare both definitions and conclude that some (fuzzy) generalization of the notion of clique is needed.
\end{abstract}

Keywords: Galois connection, Fuzzy transitive digraphs, Relational systems.

\section{Introduction}

Although introduced some time ago [24], Galois connections are still a useful tool both for theoretical and practical purposes. In fact, the underlying mathematical ideas of the theory of Formal Concept Analysis [13] is that of Galois connections; this research line has received considerable attention in the recent years, and one can find a number of publications on either its abstract generalization or its applications $[2,4,11,15,19,20,23]$.

In this paper, we continue a research line initiated in [17], where we aimed at characterizing the existence of the residual (or right part of a Galois connection) of a given mapping between sets with different structure (it is precisely this condition of different structure which makes this problem to be outside the scope of Freyd's adjoint functor theorem). Since then, we have obtained initial results in several frameworks: for instance, in [18], given a mapping from a (pre-)ordered set $\left(A, \leq_{A}\right)$ into an unstructured set $B$, we characterized the problem of completing the structure of $B$, i.e., defining a suitable (pre-)ordering relation $\leq_{B}$ on $B$, such that there exists a mapping such that the pair of mappings forms an isotone Galois connection (or adjunction) between the (pre-)ordered sets $\left(A, \leq_{A}\right)$ and $\left(B, \leq_{B}\right)$.

The initial steps for the extension to the fuzzy framework were done in [16], in which the construction of the right adjoint was done in terms of closure operators associated to Galois connection; this approach was later, completed in [5] by considering the corresponding problem for a function between a fuzzy preposet $\left(A, \rho_{A}\right)$ and an unstructured $B$; moreover, this work was recently extended in [6], by considering that equality is expressed by a fuzzy equivalence relation, so that the problem considers a mapping between a fuzzy preordered structure $\left(A, \approx_{A}, \rho_{A}\right)$ and a fuzzy structure $\left(B, \approx_{B}\right)$. These two papers satisfactorily extend the problem to the fuzzy case in both the domain and range of the Galois connection but, in both cases, the components of the Galois connection are (crisp) functions. Hence, the next logical extension is to consider the possibility that those components are actually fuzzy functions. In our opinion the term 'fuzzy Galois connection' [3, 27], should be reserved for the case in which the involved mappings are actually fuzzy mappings.

Our first attempt to obtain a properly fuzzy notion of Galois connection was to go back to the crisp case and consider a suitable relational generalization of Galois connection, one in which the domain and range are just sets endowed with arbitrary relations and whose components are (proper) relations, and this is the content of [7]. It is worth noting that, in order to preserve the existing construction via closures, we needed to provide a relational definition of Galois connection which allows the composition of the two components of the connection, and this is something that is not guaranteed by some of the relational extensions of Galois connection that can be found in the literature.

In this paper, we give the first steps towards a for- 
mal definition of fuzzy relational Galois connection between fuzzy sets with arbitrary fuzzy transitive relations (fuzzy T-digraphs), where the two components of the connection are fuzzy relations. To this end we consider, on the one hand, our definition of relational Galois connection between T-digraphs in the crisp case; and, on the other hand, our definition of fuzzy relational Galois connection between fuzzy preorders. We compare both definitions and conclude that some (fuzzy) generalization of the notion of clique is needed.

\section{Preliminary definitions}

We consider the usual framework of (crisp) relations. Namely, a binary relation $\mathcal{R}$ between two sets $A$ and $B$ is a subset of the Cartesian product $A \times B$ and it can be also seen as a (multivalued) function $\mathcal{R}$ from the set $A$ to the powerset $2^{B}$. For an element $(a, b) \in R$, it is said that $a$ is related to $b$ and denoted $a \mathcal{R} b$.

Given a binary relation $\mathcal{R} \subseteq \mathcal{A} \times \mathcal{B}$, the afterset $a^{\mathcal{R}}$ of an element $a \in A$ is defined as $\{b \in B: a \mathcal{R} b\}$.

As our Galois connections are intended to be defined between preordered structures, firstly, we will recall several forms to lift a preorder to the powersets.

Given $A$ an arbitrary set and a preorder relation $\leq$ defined over $A$, it is possible to lift $\leq$ to the powerset $2^{A}$ by defining

$$
\begin{aligned}
& X \ll Y \Longleftrightarrow \forall x \in X \exists y \in Y \text { such that } x \leq y \\
& X \Subset Y \quad \Longleftrightarrow \forall y \in Y \exists x \in X \text { such that } x \leq y
\end{aligned}
$$

We will use the term powering to refer to the lifting of a preorder to the powerset; thus, both $\Subset$ and $\ll$ above are powerings of $\leq$.

Note that the two relations defined above are actually preorder relations, specifically those used in the construction of the, respectively, Hoare and Smyth powerdomains.

Naturally, each of the extensions above induces a particular notion of isotony, inflation, etc. For instance, given two preordered sets $(A, \leq)$ and $(B, \leq),{ }^{1}$ a binary relation $\mathcal{R} \subseteq A \times B$ is said to be:

- $\Subset$-isotone if $a_{1} \leq a_{2}$ implies $a_{1}^{\mathcal{R}} \Subset a_{2}^{\mathcal{R}}$, for all $a_{1}, a_{2} \in \operatorname{dom}(\mathcal{R})$

- $\Subset$-antitone if $a_{1} \leq a_{2}$ implies $a_{2}^{\mathcal{R}} \Subset a_{1}^{\mathcal{R}}$, for all $a_{1}, a_{2} \in \operatorname{dom}(\mathcal{R})$.

A binary relation $\mathcal{R} \subseteq A \times A$ is said to be:

\footnotetext{
${ }^{1}$ Notice that, as usual, we use the same symbol to denote both binary relations which need not be equal.
}

- $\Subset$-inflationary if $\{a\} \Subset a^{\mathcal{R}}$, for all $a \in \operatorname{dom}(\mathcal{R})$;

- $\Subset$-deflationary if $a^{\mathcal{R}} \Subset\{a\}$, for all $a \in \operatorname{dom}(\mathcal{R})$.

- $\Subset$-idempotent if $a^{\mathcal{R} \circ \mathcal{R}} \Subset a^{\mathcal{R}}$ and $a^{\mathcal{R}} \Subset a^{\mathcal{R} \circ \mathcal{R}}$, for all $a \in \operatorname{dom}(\mathcal{R})$.

We use the prefix to distinguish the powering used in the different definitions.

Traditionally, a Galois connection is understood as a pair of antitone mappings whose compositions are both inflationary, and has a number of different alternative characterizations. In our generalized relational setting, we have a wide choice for characterization used to give the formal definition, and also to the different notions of antitonicity and inflation (depending on the powering), or even the relational composition to be used.

In this paper, we will work with the usual notion of relational composition. Let $\mathcal{R}$ be a binary relation between $A$ and $B$ and $\mathcal{S}$ be a binary relation between $B$ and $C$. The composition of $\mathcal{R}$ and $\mathcal{S}$ is defined as follows

$$
\mathcal{R} \circ \mathcal{S}=\{(x, z) \in A \times C \mid \exists b \in B \text { with } x \mathcal{R} b \text { and } b \mathcal{S} z\}
$$

Observe that for an element $a \in A$, the afterset $a^{\mathcal{R} \circ \mathcal{S}}$ can be written as $\bigcup_{b \in a^{\mathcal{R}}} b^{\mathcal{S}}$.

\subsection{Fuzzy preliminaries}

Given a complete residuated lattice $\mathbb{L}=(L, \otimes, \Rightarrow)$, an $\mathbb{L}$-fuzzy set is a mapping from the universe set to the membership values structure $X: U \rightarrow L$ where $X(u)$ means the degree in which $u$ belongs to $X$.

An $\mathbb{L}$-fuzzy binary relation on $U$ is an $\mathbb{L}$-fuzzy subset of $U \times U$, that is $R_{U}: U \times U \rightarrow L$, and it is said to be:

- Reflexive if $R_{U}(a, a)=\top$ for all $a \in U$.

- $\otimes$-Transitive if $R_{U}(a, b) \otimes R_{U}(b, c) \leq R_{U}(a, c)$ for all $a, b, c \in U$.

From now on, when no confusion arises, we will omit the prefix "LL-".

Definition 1. A fuzzy T-digraph is a pair $\mathbb{A}=$ $\left\langle A, \rho_{A}\right\rangle$ in which $\rho_{A}$ is a $\otimes$-transitive fuzzy relation on $A$.

Definition 2. $A$ fuzzy preposet is a pair $\mathbb{A}=\left\langle A, \rho_{A}\right\rangle$ in which $\rho_{A}$ is a reflexive and $\otimes$-transitive fuzzy relation on $A$.

Definition 3. A fuzzy relation $\mu: A \times B \rightarrow L$ is said to be total if and only if or all $a \in A$ there exists $b \in B$ satisfying that $\mu(a, b)=\top$. 


\section{A relational extension of Galois connections}

Our goal in this work is to define the notion of Galois connection as a pair of relations between sets with the least possible structure. As all of the results make use in some way of the transitive property, although we could work with arbitrary relations and use its transitive closure, in order to improve the readability of the results we will assume that the relations are transitive from the beginning.

Hereinafter, we refer to a couple $(A, \tau)$, where $\tau \subseteq$ $A \times A$, as a digraph, and when $\tau$ is transitive we call T-digraph.

A T-digraph $(A, \tau)$ will be often represented as $\mathbb{A}$, to refer to the underlying set $A$, the accompanying relation will be written $\tau$ whenever no ambiguities arise, similarly to what happens with ordered sets in which the ordering relation are usually called $\leq$.

It is worth to remark that the powerings $\Subset$ and $\ll$ can be defined for any relation $\tau$ not necessarily being a preorder relation.

A well-known characterization of a Galois connection $(f, g)$ between two posets is the so-called Galois condition

$$
a \leq g(b) \Longleftrightarrow b \leq f(a)
$$

As stated above, in our general framework there are several possible choices, which we will distinguish by using the corresponding prefix. For instance, given two relations $\mathcal{R}$ and $\mathcal{S}$, the $\ll$-Galois condition is

$$
\{a\} \ll b^{\mathcal{S}} \quad \Longleftrightarrow \quad\{b\} \ll a^{\mathcal{R}}
$$

In [10], we studied the properties of the different extensions obtained in terms of the powerings $\ll$ and $\Subset$ used in the corresponding Galois condition. Later, in [7], we focussed our attention on another desirable characterization, the definition of Galois connection in terms of closures. We introduce below the corresponding relational extension of the notion of closure operator.

Definition 4. Given a T-digraph $(A, \tau)$, a powering $*$ of $\leq$, and $\mathcal{C} \subseteq A \times A$, we say that $\mathcal{C}$ is a*closure relation, if $\mathcal{C}$ is $*$-isotone, $*$-inflationary, and *-idempotent.

Definition 5. A relational Galois connection between two $T$-digraphs $\mathbb{A}$ and $\mathbb{B}$ is a pair of relations $(\mathcal{R}, \mathcal{S})$ where $\mathcal{R} \subseteq A \times B$ and $\mathcal{S} \subseteq B \times A$ such that the following properties hold:

i. $\mathcal{R}$ and $\mathcal{S}$ are $\Subset$-antitone. ii. $\mathcal{R} \circ \mathcal{S}$ and $\mathcal{S} \circ \mathcal{R}$ are $\Subset$-inflationary.

We can see below an example in which both $\mathcal{R}$ and $\mathcal{S}$ are proper (non-functional) relations.

Example 1. Consider $\mathbb{A}=(A, \tau)$ where $A=\{1,2,3\}$ and $\tau$ is the transitive relation $\{(1,2),(1,3),(2,2),(2,3),(3,2),(3,3)\}$. The pair of relations $(\mathcal{R}, \mathcal{S})$ given by the tables below constitutes a relational Galois connection between $\mathbb{A}$ and $\mathbb{A}$.

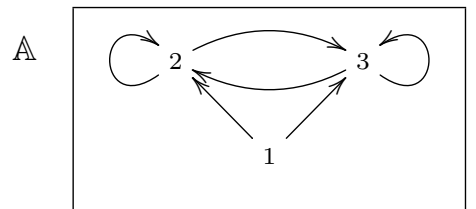

\begin{tabular}{c|c}
$x$ & $x^{\mathcal{R}}$ \\
\hline 1 & $\{2,3\}$ \\
2 & $\{2\}$ \\
3 & $\{3\}$
\end{tabular}

\begin{tabular}{c|c}
$x$ & $x^{\mathcal{S}}$ \\
\hline 1 & $\{2,3\}$ \\
2 & $\{2\}$ \\
3 & $\{2,3\}$
\end{tabular}

The interesting part is that the $\Subset$ powering guarantees that both compositions in a relational Galois connection lead to $\Subset$-closure relation. Formally, we have the following result:

Theorem 1. Given a relational Galois connection $(\mathcal{R}, \mathcal{S})$ between $\mathbb{A}$ and $\mathbb{B}$, we have that $\mathcal{R} \circ \mathcal{S}$ and $\mathcal{S} \circ \mathcal{R}$ are $\Subset$-closure relations.

\section{Comparison with other approaches}

Essential Galois bonds between formal contexts were introduced by Xia [26] in the framework of FCA, and are important for our work in that its components are relations. This definition was later renamed as relational Galois connection in [14], where a unifying language was provided in order to cope with similar attempts, previously given by Domenach and Leclerc [12] and by Wille [25].

Recall that for ordered sets $\left(P, \leq_{1}\right)$ and $\left(Q, \leq_{2}\right)$ to state that $(\varphi, \psi)$ is a Galois connection between the contexts $\left(P, P, \leq_{1}\right)$ and $\left(Q, Q, \leq_{2}\right)$ translates to the usual Galois condition

$$
x \leq_{1} \psi(y) \Longleftrightarrow y \leq_{2} \varphi(x)
$$

The first approach natural notion of Galois connection between arbitrary contexts $(G, M, I)$ and $(H, N, J)$ is given in [14] as a pair of mappings $(\varphi, \psi)$ where $\varphi: G \rightarrow N$ and $\psi: H \rightarrow M$, satisfying the corresponding Galois condition, namely,

$$
g I \psi(h) \Longleftrightarrow h J \varphi(g)
$$


Then, a further (and natural) generalisation in which the pair of mappings are replaced by a pair of arbitrary relations $\Phi \subseteq G \times N$ and $\Psi \subseteq H \times M$ should include the corresponding relational version of the Galois condition, which is called the relational Galois condition:

$$
g I h^{\Psi} \Longleftrightarrow h J g^{\Phi}
$$

This condition by itself is not strong enough in the relational framework and, hence, Xia introduced a certain optimality of $\Phi$ and $\Psi$. This condition was simplified in [14] in terms of intents of the corresponding contexts as follows:

Definition 6. A relational Galois connection between two contexts $(G, M, I)$ and $(H, N, J)$ is a pair of relations $(\Phi, \Psi)$ where $\Phi \subseteq G \times N$ and $\Psi \subseteq H \times M$ satisfying

- $g I h^{\Psi} \Longleftrightarrow h J g^{\Phi}$ for all $g \in G, h \in H$.

- $g^{\Phi}$ is an intent of $(H, N, J)$ and $h^{\Psi}$ is an intent of $(G, M, I)$.

This definition, as stated in [14, Lemma 1], leads back to the original definition of Galois connection between lattices, in that every classical Galois connection between the concept lattices $\underline{\mathfrak{B}}(G, M, I)$ and $\underline{\mathfrak{B}}(H, N, J)$ of two contexts $(G, M, I)$ and $(H, N, J)$ respectively, defines a relational Galois connection between the contexts $(G, M, I)$ and $(H, N, J)$ and vice versa, being this correspondence one-to-one.

The following two examples show that Definition 6 of relational Galois connection does not imply nor is implied by our definition.

Example 2. Let $\mathbb{A}$ and $\mathbb{B}$ be the $T$-digraphs shown below, and $\mathcal{R} \subseteq A \times B$ and $\mathcal{S} \subseteq B \times A$ the relations defined as follows:

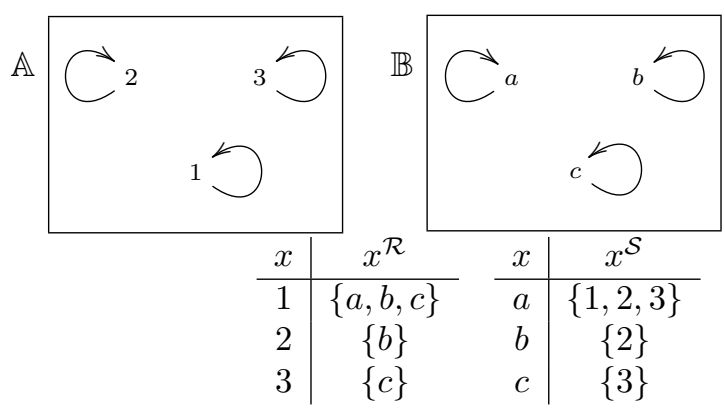

It is easy to check that $(\mathcal{R}, \mathcal{S})$ verifies Definition 6 but does not satisfy our definition, because $\mathcal{R} \circ \mathcal{S}$ fails to be inflationary in element 1. As a consequence, $\mathcal{R} \circ \mathcal{S}$ is not a closure relation.

Example 3. Let $\mathbb{A}$ and $\mathbb{B}$ be the T-digraphs shown below, and $\mathcal{R} \subseteq A \times B$ and $\mathcal{S} \subseteq B \times A$ the relations defined as follows:

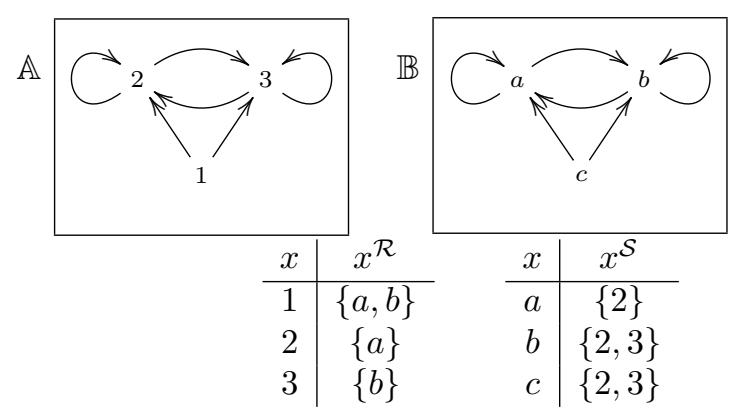

The pair $(\mathcal{R}, \mathcal{S})$ constitutes a relational Galois connection between $\left(A, \tau_{A}\right)$ and $\left(B, \tau_{B}\right)$ by our definition. However, it does not verify Definition 6 . For instance, $2^{\mathcal{R}}=\{a\}$ is not an intent since $2^{\mathcal{R} \tau^{-1} \tau}=\{a, b\} \neq 2^{\mathcal{R}}$.

\section{Characterization of relational Galois connections}

Having in mind the different characterizations of classical Galois connections between posets in terms of the Galois condition, the definition of a relational Galois connection (Definition 5) might also be equivalent to the corresponding Galois condition, namely:

$$
\{a\} \Subset b^{\mathcal{S}} \Longleftrightarrow\{b\} \Subset a^{\mathcal{R}}, \forall a \in A, b \in B
$$

In order to characterize the notion of relational Galois connection, we will introduce an alternative powering of a relation $\tau$ to the powersets:

$$
X \propto Y \Longleftrightarrow x \tau y \text { for all } x \in X \text { and for all } y \in Y
$$

Remark 1. Note that $\propto$ need not be either reflexive nor transitive. Nevertheless, for a T-digraph it satisfies the following weakened version of transitivity:

For any $Y \neq \varnothing$, if $X \propto Y$ and $Y \propto Z$, then $X \propto Z$.

We will see that the Galois condition (1), together with a certain technical condition somewhat related to the reflexivity of $\propto$, is equivalent to the definition of a relational Galois connection.

Definition 7. Let $\mathbb{A}$ be a T-digraph and $X \subseteq A$. It is said that $X$ is a clique if $X \propto X$.

Now, we will give the following technical result.

Lemma 1. Let $\mathbb{A}$ be a T-digraph and $x \in X \subseteq A$. If $X$ is a clique then, for all $Y \subseteq A$, the following statements hold:

i. $Y \propto\{x\}$ implies $Y \propto X$.

ii. $\{x\} \propto Y$ implies $X \propto Y$.

iii. $X \Subset Y$ if and only if $X \propto Y$. 
Note that there exists a tight relation between $\Subset$ and $\propto$, since for all $x \in A$ and all $Y \subseteq A$ we have that

$$
\{x\} \Subset Y \quad \Longleftrightarrow \quad\{x\} \propto Y
$$

particularly, the notions of $\Subset$-inflation and $\propto$-inflation are equivalent and, moreover, the corresponding versions of the Galois condition are also equivalent.

Our first characterization of relational Galois connections is based on the fact that the direct images of singletons should be cliques for both components of the relational Galois connection. The formal result is as follows:

Proposition 1. $(\mathcal{R}, \mathcal{S})$ is a relational Galois connection between $(A, \tau)$ and $(B, \tau)$ iff the following properties hold:

$$
\begin{aligned}
& \text { i. }\{a\} \Subset b^{\mathcal{S}} \text { iff }\{b\} \Subset a^{\mathcal{R}} \text { for all } a \in A \text {, and } b \in B \text {. } \\
& \text { ii. } a^{\mathcal{R}} \text { and } b^{\mathcal{S}} \text { are cliques for all } a \in A \text { and } b \in B \text {. }
\end{aligned}
$$

The next result shows that our definition of $\Subset$-based relational Galois connection coincides exactly with the corresponding $\propto$-version.

Proposition 2. $(\mathcal{R}, \mathcal{S})$ is a relational Galois connection between $(A, \tau)$ and $(B, \tau)$ iff the following properties hold:

i. $\mathcal{R}$ and $\mathcal{S}$ are $\propto$-antitone.

ii. $\mathcal{R} \circ \mathcal{S}$ and $\mathcal{S} \circ \mathcal{R}$ are $\propto$-inflationary.

\section{Towards fuzzy relational Galois connections}

In this section, we adapt the definition of relational fuzzy adjunction given in [9] between fuzzy preposets to the case of Galois connections.

Definition 8. Let $\left\langle A, \rho_{A}\right\rangle$ and $\left\langle B, \rho_{B}\right\rangle$ be fuzzy preposets and $\mu: A \times B \rightarrow \mathbb{L}$ and $\nu: B \times A \rightarrow \mathbb{L}$ be total fuzzy relations. The pair $(\mu, \nu)$ is said to be $a$ relational fuzzy Galois connection between $\left\langle A, \rho_{A}\right\rangle$ and $\left\langle B, \rho_{B}\right\rangle$ if the following conditions hold for all $a_{1}, a_{2} \in A$ and $b_{1}, b_{2} \in B$ :

$$
\begin{aligned}
\text { i) } \rho_{A}\left(a_{1}, a_{2}\right) \otimes \mu\left(a_{1}, b_{1}\right) \otimes \nu\left(b_{2}, a_{2}\right) \leq \rho_{B}\left(b_{2}, b_{1}\right) \text {. } \\
\text { ii) } \rho_{B}\left(b_{2}, b_{1}\right) \otimes \mu\left(a_{1}, b_{1}\right) \otimes \nu\left(b_{2}, a_{2}\right) \leq \rho_{A}\left(a_{1}, a_{2}\right) \text {. }
\end{aligned}
$$

In order to study the properties of relational fuzzy Galois connections, we need to adapt the well-known notions of antitone and inflationary mapping.

Definition 9. Let $\left\langle A, \rho_{A}\right\rangle$ and $\left\langle B, \rho_{B}\right\rangle$ be fuzzy preposets. A fuzzy relation $\mu: A \times B \rightarrow \mathbb{L}$ is said to be antitone if $\rho_{A}\left(a_{1}, a_{2}\right) \otimes \mu\left(a_{1}, b_{1}\right) \otimes \mu\left(a_{2}, b_{2}\right) \leq \rho_{B}\left(b_{2}, b_{1}\right)$ for all $a_{1}, a_{2} \in A$ and $b_{1}, b_{2} \in B$.
Definition 10. Let $\left\langle A, \rho_{A}\right\rangle$ be a fuzzy preposet. A fuzzy relation $\mu: A \times A \rightarrow \mathbb{L}$ is said to be inflationary if $\mu\left(a_{1}, a_{2}\right) \leq \rho_{A}\left(a_{1}, a_{2}\right)$ for all $a_{1}, a_{2} \in A$.

The following theorem gives a characterization of relational fuzzy Galois connections between preposets.

Theorem 2 (See [8]). Let $\left\langle A, \rho_{A}\right\rangle$ and $\left\langle B, \rho_{B}\right\rangle$ be fuzzy preposets and $\mu: A \times B \rightarrow \mathbb{L}$ and $\nu: B \times A \rightarrow \mathbb{L}$ be total fuzzy relations. Then, $(\mu, \nu)$ is a relational fuzzy Galois connection if and only if $\mu$ and $\nu$ are antitone, and both $\nu \circ \mu$ and $\mu \circ \nu$ are inflationary.

Now, we translate the conditions in the previous Definition 8 to the crisp case.

Definition 11. Let $\mathbb{A}$ and $\mathbb{B}$ be two T-digraphs, $\mathcal{R} \subseteq$ $A \times B$ and $\mathcal{S} \subseteq B \times A$. The pair of relations $(\mathcal{R}, \mathcal{S})$ satisfies the F-Galois condition if the two following conditions hold, for all $a \in A$ and $b \in B$ :

$$
\begin{aligned}
& \text { i. } a \ll b^{\mathcal{S}} \text { implies } b \Subset a^{\mathcal{R}} \text {. } \\
& \text { ii. } b \ll a^{\mathcal{R}} \text { implies } a \Subset b^{\mathcal{S}} \text {. }
\end{aligned}
$$

The following result links the notion of crisp relational Galois connection presented in Definition 5 to the notion of fuzzy relational Galois connection given in Definition 8 .

Theorem 3. If $\mathbb{A}$ and $\mathbb{B}$ are preorders, the following conditions are equivalent:

i. $(\mathcal{R}, \mathcal{S})$ satisfies F-Galois condition.

ii. $(\mathcal{R}, \mathcal{S})$ satisfies $\Subset-$ Galois condition and $a^{\mathcal{R}}$ and $b^{\mathcal{S}}$ are cliques, for all $a \in A$ and $b \in B$.

iii. $(\mathcal{R}, \mathcal{S})$ is a relational Galois connection.

Proof. Notice that (ii) and (iii) are equivalent by Proposition 1. Let us prove now that (i) and (ii) are equivalent.

Let us prove first that the F-Galois condition implies the $\Subset$-Galois condition. For all $a \in A$ and $b \in B$, if $a \Subset b^{\mathcal{S}}$ then, as $\mathcal{S}$ is total, $a \ll b^{\mathcal{S}}$, and by (i), $b \Subset a^{\mathcal{R}}$ or, equivalently, $b \Subset a^{\mathcal{R}}$. The other implication is similarly proved. For any $b \in a^{\mathcal{R}}$, as $\tau$ is reflexive, we have that $b \tau b$, hence $b \ll a^{\mathcal{R}}$, which implies, by hypothesis, that $a \Subset b^{\mathcal{S}}$. Since $\mathcal{S}$ is total, this also implies $a \ll b^{\mathcal{S}}$. Again by hypothesis, this leads to $b \Subset a^{\mathcal{R}}$, which proves that $a^{\mathcal{R}}$ is a clique. The proof that $b^{\mathcal{S}}$ is a clique is similar.

Conversely, assume that the $\Subset$-Galois condition holds, and that $a^{\mathcal{R}}$ and $b^{\mathcal{S}}$ are cliques, for all $a \in A$ and $b \in B$. If $a \ll b^{\mathcal{S}}$, there exists $x \in b^{\mathcal{S}}$ such that $a \tau x$. As $b^{\mathcal{S}}$ is a clique, by Lemma 1 , we have that $a \Subset b^{\mathcal{S}}$, and this implies, by hypothesis, that $b \Subset a^{\mathcal{R}}$, that is, $b \Subset a^{\mathcal{R}}$, proving the first item of the F-Galois condition. The proof of the second item is similar. 
The following example shows that the Galois condition does not imply the F-Galois condition.

Example 4. Consider the T-digraph $\mathbb{A}$ and the relations $\mathcal{R}$ and $\mathcal{S}$ depicted below.

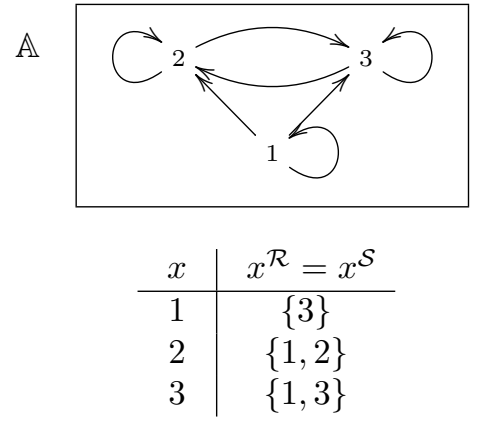

It is routine to prove that $(\mathcal{R}, \mathcal{S})$ verifies the Galois condition, but it does not verify the F-Galois condition, because, for instance, $2 \ll 3^{\mathcal{S}}$ but $3 \notin 2^{\mathcal{R}}$.

The following example shows that the F-Galois condition is not enough to have a relational Galois connection in the non-reflexive case.

Example 5. Consider the T-digraphs $\mathbb{A}, \mathbb{B}$, and the relations $\mathcal{R}$ and $\mathcal{S}$ depicted below.
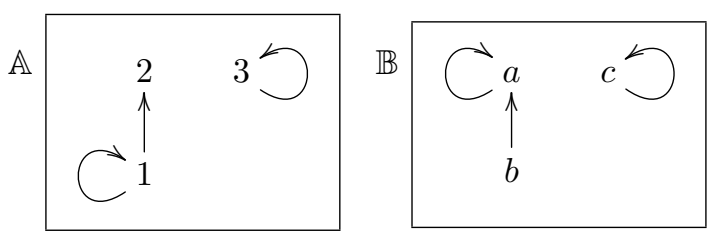

\begin{tabular}{c|ccc|c}
$x$ & $x^{\mathcal{R}}$ & & $x$ & $x^{\mathcal{S}}$ \\
\hline 1 & $\{a\}$ & & $a$ & $\{1\}$ \\
2 & $\{b\}$ & $b$ & $\{1,2\}$ \\
3 & $\{c\}$ & $c$ & $\{3\}$
\end{tabular}

It is easy to check that $(\mathcal{R}, \mathcal{S})$ verifies the F-Galois condition, but it is not a relational Galois connection, because $\{1\} \in 2^{\mathcal{R} \circ \mathcal{S}}$, while $2 \nmid 1$, which contradicts $\{2\} \Subset_{A} 2^{\mathcal{R} \circ \mathcal{S}}$.

\section{Conclusions and future work}

We presented the first steps towards a formal definition of fuzzy relational Galois connection between fuzzy T-digraphs, where the two components of the connection are fuzzy relations. A comparison between our definition of relational Galois connection between T-digraphs in the crisp case, and our definition of fuzzy relational Galois connection between fuzzy preorders has been done, reaching the conclusion that some (fuzzy) generalization of the notion of clique is needed. Moreover, we compared the (crisp) approach with similar ones presented in the framework of FCA.
Concerning future work, we are working on a suitable definition of fuzzy clique which would allow to define the notion of fuzzy relational Galois connection in terms of the Galois condition, adding this notion of clique. This would open the door towards the search for a characterization of the existence of residual in a fuzzy setting given a fuzzy relation between unbalanced fuzzy structures; Moreover, this study also enables a new approach to FCA, and could also have implications in further advances in the study of generalized Chu correspondences, which will pave the way to use the approach given in [21] to analyze more structures related to quantum logics, such as those in $[1,22]$.

\section{Acknowledgement}

Partially supported by the Spanish research projects TIN15-70266-C2-P-1, PGC2018-095869-B-I00 and TIN2017-89023-P of the Science and Innovation Ministry of Spain and the European Social Fund.

\section{References}

[1] S. Abramsky. Big toy models: representing physical systems as Chu spaces. Synthese, 186(3):697-718, 2012.

[2] L. Antoni, S. Krajči, and O. Krídlo. Representation of fuzzy subsets by Galois connections. Fuzzy Sets and Systems 326:52-68, 2017.

[3] R. Bělohlávek. Fuzzy Galois connections. Mathematical Logic Quarterly, 45(4):497-504, 1999

[4] P. Butka, J. Pócs, and J. Pócsova: Isotone Galois Connections and Generalized One-Sided Concept Lattices. Proc. of Multimedia and Network Information Systems (MISSI), 151-160, 2018.

[5] I.P. Cabrera, P. Cordero, F. García-Pardo, M. Ojeda-Aciego, and B. De Baets. On the construction of adjunctions between a fuzzy preposet and an unstructured set. Fuzzy Sets and Systems 320:81-92, 2017.

[6] I.P. Cabrera, P. Cordero, F. García-Pardo, M. Ojeda-Aciego, and B. De Baets. Adjunctions between a fuzzy preposet and an unstructured set with underlying fuzzy equivalence relations. IEEE Tr. on Fuzzy Systems 26(3):1274-1287, 2018.

[7] I.P. Cabrera, P. Cordero, E. Muñoz-Velasco and M. Ojeda-Aciego. A relational extension of Galois Connections. In Proc. of Intl Conf on Formal Concept Analysis, 2019. Lect. Notes in Artificial Intelligence 11511. https://doi .org/10.1007/978-3-030-21462-3_19 
[8] I.P. Cabrera, P. Cordero, and M. Ojeda-Aciego. On fuzzy relations, functional relations, and adjunctions. In Proc. of Foundations of Computational Intelligence, FOCI, 2016.

[9] I.P. Cabrera, P. Cordero, and M. Ojeda-Aciego. Towards relational fuzzy adjunctions. In Proc. of 2017 IEEE International Conference on Fuzzy Systems (FUZZ-IEEE)

[10] I.P. Cabrera, P. Cordero, and M. Ojeda-Aciego. Relation-based Galois connections: towards the residual of a relation. In Proc. of Computational and Mathematical Methods in Science and Engineering (CMMSE'17), 2017.

[11] J.T. Denniston, A. Melton, and S.E. Rodabaugh. Formal Contexts, Formal Concept Analysis, and Galois Connections. Electr. Proc. on Theoretical Computer Science 129: 105-120, 2013.

[12] F. Domenach and B. Leclerc. Biclosed binary relations and Galois connections. Order 18(1):89$104,2001$.

[13] B. Ganter and R. Wille. Formal concept analysis. Springer-Verlag. 1999

[14] B. Ganter. Relational Galois connections Lect. Notes in Computer Science 4390:1-17, 2007

[15] F. García, I.P. Cabrera, P. Cordero, and M. Ojeda-Aciego. On Galois connections and soft computing. Lect. Notes in Computer Science. 7903:224-235, 2013.

[16] F. García-Pardo, I.P. Cabrera, P. Cordero, and M. Ojeda-Aciego. On closure systems and adjunctions between fuzzy preordered sets. Lect. Notes in Computer Science, 9113:114-127, 2015.

[17] F. García, I.P. Cabrera, P. Cordero, M. OjedaAciego, F. Rodriguez. On the Existence of Isotone Galois Connections between Preorders. Lect. Notes in Computer Science 8478:67-79, 2014.
[18] F. García, I.P. Cabrera, P. Cordero, M. OjedaAciego, F. Rodriguez. On the definition of suitable orderings to generate adjunctions over an unstructured codomain. Information Sciences, 286:173-187, 2014.

[19] J. Gutiérrez-García, H. Lai, and L. Shen. Fuzzy Galois connections on fuzzy sets. Fuzzy Sets and Systems 352: 26-55, 2018

[20] E. Jeřábek. Galois connection for multipleoutput operations. Algebra Universalis 79:17, 2018.

[21] O. Krídlo and M. Ojeda-Aciego. Formal concept analysis and structures underlying quantum logics. Comm. in Computer and Information Science 853:574-584, 2018.

[22] O. Krídlo and M. Ojeda-Aciego. Relating Hilbert-Chu correspondences and big toy models for quantum mechanics. In Proc. of European Symposium on Computational Intelligence and Mathematics, 2018.

[23] V. Kursitys and D.I. Ignatov. Understanding Collaborative Filtering with Galois Connections. Proceedings of What can FCA do for AI? (FCA4AI@IJCAI), 127-143, 2018.

[24] O. Ore. Galois Connexions. Transactions of the American Mathematical Society, 55(3):493-513, 1944.

[25] R. Wille. Subdirect product constructions of concept lattices. Discrete Mathematics 63:305313, 1987.

[26] W. Xia. Morphismen als formale BegriffeDarstellung und Erzeugung. Verlag Shaker 1993.

[27] W. Yao and L.-X. Lu. Fuzzy Galois connections on fuzzy posets. Mathematical Logic Quarterly, 55(1):105-112, 2009. 